\title{
Inquérito epidemiológico em população idosa (parte II): saúde bucal, ansiedade, depressão, estresse e uso de medicamentos
}

\author{
Epidemiological survey in elderly people (II): oral health, anxiety, depression,
} stress, and drug utilization

\author{
Rafael Menezes-Silva ${ }^{1}$, Dhelfeson Willya Douglas de Oliveira², Paulo Cesar Brambilla Biscaro ${ }^{3}$, \\ Natália Pinheiro Orti ${ }^{4}$, Ana Clara Sá-Pinto ${ }^{5}$, Maria Letícia Ramos-Jorge ${ }^{5}$ \\ ${ }^{1}$ Departamento de Dentística, Endodontia e Materiais Odontológicos, Faculdade de Odontologia de Bauru da Universidade de São Paulo (FOB-USP). Bauru, SP. \\ 2 Departamento de Periodontia, Faculdade de Odontologia da Universidade Federal de Minas Gerais (UFMG). Belo Horizonte, MG. \\ Clínica privada de Geriatria. Bauru, SP \\ ${ }^{4}$ Instituto de Análise do Comportamento (IACB). Bauru, SP. \\ ${ }^{5}$ Departamento de Odontologia, Universidade Federal dos Vales do Jequitinhonha e Mucuri (UFVJM). Diamantina, MC.
}

\section{RESUMO}

Objetivos: Investigar as correlações existentes entre saúde bucal, ansiedade, depressão, estresse, alterações cognitivas e uso de medicamentos em idosos.

Métodos: Os dados foram coletados em domicílio, por meio de questionários sobre dados sociodemográficos e uso de medicações; exames bucais para investigar o índice de dentes cariados, perdidos e obturados; e instrumentos para avaliar a presença de distúrbios psicossociais Os seguintes instrumentos foram aplicados: Mini Exame do Estado Mental (alterações cognitivas), Escala de Katz (grau de dependência), Inventário de Depressão de Beck, Escala de Depressão Geriátrica (depressão), Inventário de Ansiedade de Beck (ansiedade) e Inventário de Sintomas de Stress para adultos de Lipp (estresse). Também se investigou a quantidade de remédios usados. Foi realizada análise descritiva e teste de correlação de Spearman.

Resultados: Foram incluídos 37 idosos com idade média de 73,41 anos. O índice CPO-D médio foi 25,38. Dos 37 idosos, $21,6 \%$ foram classificados com depressão leve/moderada, $45,9 \%$ apresentavam ansiedade, $78,4 \%$ tinham comprometimento cognitivo e $54,1 \%$ tinham polifarmácia menor. Foram encontradas correlações estatisticamente significativas de forma positiva e moderada entre idade e saúde bucal; de forma negativa moderada entre idade e alterações cognitivas; e de forma positiva forte entre depressão e estresse resistência e entre ansiedade e estresse resistência.

Conclusões: Saúde bucal, ansiedade, depressão, estresse e medicação foram fatores correlacionados na amostra de idosos estudada. DESCRITORES: saúde bucal; ansiedade; depressão; uso de medicamentos.

\section{ABSTRACT}

Aims: To investigate the correlation between oral health, anxiety, depression, stress, cognitive impairment, and use of medications in the elderly. Methods: Data were collected using household survey questionnaires on sociodemographic variables and use of medications; oral examinations for detection of decayed, missing, and filled teeth; and questionnaires to verify the presence of psychosocial disorders. The following instruments were applied: mini-mental state examination (cognitive impairment), Katz scale (index of independence), Beck depression inventory, geriatric depression scale (depression), Beck anxiety inventory (anxiety), and Lipp's stress symptom inventory for adults (stress). The quantity of medication used was also investigated. A descriptive analysis and Spearman's correlation test were performed.

Results: Thirty-seven elderly individuals, mean age of 73.41 years, were included in the study. The mean DMFT index was 25.38 . Mild to moderate depression was detected in $21.6 \%$, anxiety in $45.9 \%$, cognitive impairment in $78.4 \%$, and lower polypharmacy rates in $54.1 \%$. Age and oral health showed a moderate statistically significant positive correlation; age and cognitive impairment revealed a moderate negative correlation; and there was a strong and positive correlation between depression and the resistance stage of stress and between anxiety and the resistance stage of stress

Conclusions: There was correlation between oral health, anxiety, depression, stress, and medication in the investigated elderly sample.

KEY WORDS: oral health; anxiety; depression; drug utilization.

Recebido: setembro, 2015

Aceito: janeiro, 2016

Este artigo está licenciado sob forma de uma licença Creative Commons Atribuição 4.0 Internacional, que permite uso irrestrito, distribuição e reprodução 
Abreviaturas (e finalidades dos instrumentos utilizados): CPO-D, Índice de Dentes Cariados, Perdidos e Obturados (saúde bucal); MEEM, Mini Exame do Estado Mental (alterações cognitivas); Escala Katz (independência funcional); EDG, Escala de Depressão Geriátrica (depressão); BDI, Inventário de Depressão de Beck (depressão); BAI, Inventário de Ansiedade de Beck (ansiedade); ISSL, Inventário de Sintomas de Stress para adultos de Lipp (estresse).

\section{INTRODUÇÃO}

O envelhecimento populacional já evidente nos países desenvolvidos vem ocorrendo no Brasil e demais países em desenvolvimento [1]. De acordo com as Nações Unidas, a população brasileira com até 44 anos de idade deverá decrescer entre 2000 e 2050 , enquanto a população entre 65 e 74 anos irá crescer $321 \%$ no mesmo período, sendo que para pessoas com 75 anos ou mais de idade o crescimento esperado é de $622 \%$ [2]. A prevalência de doenças ligadas ao envelhecimento, sejam crônicas ou não, cresce proporcionalmente. Dentre elas, os transtornos mentais mostram-se relevantes tanto pela grande incidência como por comprometerem a qualidade de vida. Dentre os diagnósticos mais prevalentes estão o transtorno depressivo, acometendo de 4,8 a $14,6 \%$ dos idosos, o transtorno de ansiedade isolado em até $4 \%$ e o declínio cognitivo em 5,1 a 19\%. O prejuízo na qualidade de vida está relacionado principalmente com o comprometimento da independência, perda de interesse em atividades previamente prazerosas e isolamento, com prejuízo das relações sociais $[3,4]$.

O transtorno depressivo apresenta-se de forma relevante entre a população idosa, constituindo um problema de saúde pública [5]. Estima-se que a prevalência de depressão em idosos seja de 15\% [6], aumentando para $60 \%$ quando são institucionalizados $[7,8]$. Outro agravo comum na terceira idade é o transtorno de ansiedade que, junto com a depressão, pode estar ligado a doenças incapacitantes, insuficiência de suporte social e dificuldades nas relações interpessoais, levando a sofrimento físico e psíquico [9-11]. Estudos têm mostrado também a ocorrência de estresse na população idosa, relacionado a problemas como complicações na aposentadoria, dificuldades financeiras, morte de amigos e familiares e discriminação social [12,13]. Declínio cognitivo também é um importante aspecto a ser considerado da população idosa. As alterações cognitivas constituem um dos mais importantes aspectos que repercutem negativamente na funcionalidade das pessoas $[14,15]$.
Além das doenças relacionadas à saúde mental, comorbidades cardiovasculares, respiratórias, endócrinas e reumáticas, entre outras, também têm sua incidência aumentada com o envelhecimento, acarretando muitas vezes a necessidade do uso de múltiplos medicamentos [16-19]. A polifarmácia pode trazer consequências negativas especialmente em idosos, cujas alterações funcionais modificam a farmacocinética dos medicamentos [20].

As definições para polifarmácia são numerosas, e os critérios variam de estudo para estudo [21-24]. De acordo com uma revisão da literatura, polifarmácia é geralmente definida de duas maneiras: pelo número dos medicamentos utilizados, ou pelo uso de mais medicamentos do que os que seriam indicados clinicamente [24]. Não existe um consenso absoluto sobre qual o número de medicamentos utilizados deve ser considerado como polifarmácia. Pesquisadores têm definido polifarmácia como o uso a longo prazo de um número diário acima de dois a cinco medicamentos $[23,25,26]$.

Um estudo piloto que vem sendo realizado com idosos da cidade de Diamantina, Minas Gerais, com especial interesse na saúde bucal, detectou correlações entre índice de dentes cariados, perdidos e obturados (CPO-D), qualidade de vida e locus de controle da saúde [27]. O presente estudo constitui a continuação do inquérito epidemiológico na mesma amostra de idosos, tendo como objetivo investigar as correlações entre saúde bucal, depressão, distúrbio de ansiedade, estresse e uso de medicamentos.

\section{MÉTODOS}

Trata-se de um estudo piloto de delineamento transversal, que incluiu idosos de ambos os gêneros sem distinção de cor ou condição socioeconômica. $\mathrm{O}$ estudo foi realizado na cidade de Diamantina, Minas Gerais, a qual tem uma população total de 47.647 habitantes. O protocolo de pesquisa foi aprovado pelo Comitê de Ética em Pesquisa com Seres Humanos da Universidade Federal dos Vales do Jequitinhonha e Mucuri sob o parecer número 118/12.

Embora exista ausência de consenso e fórmula matemática para determinar o tamanho amostral de um estudo piloto, a literatura reporta entre 30 a 50 participantes como suficientes [28]. Conforme já descrito no estudo anterior [27], foi realizada amostragem probabilística pela técnica de amostragem estratificada uniforme. Para o desenvolvimento do estudo piloto, a cidade de Diamantina foi dividida em nove estratos de acordo com a cobertura das unidades 
básicas de saúde. Em cada estrato, foi obtida a lista de todos os idosos cadastrados; os idosos que seriam investigados foram selecionados por sorteio.

Os critérios de inclusão foram pessoas acima de 60 anos cadastradas nas Estratégias Saúde da Família. Foram excluídas pessoas com alterações comportamentais, hospitalizadas, institucionalizadas, ou com doenças sistêmicas graves [27].

Os dados foram coletados em domicílio, de janeiro a março de 2015, por meio de exames bucais e questionários de heteroavaliação. A equipe de trabalho de campo era formada por um examinador e por um auxiliar responsável em anotar os dados coletados e em aplicar os questionários. Foi feito um treinamento prévio com o entrevistador para que o mesmo realizasse a leitura dos itens de forma clara e objetiva, de modo a não influenciar a resposta do idoso. O questionário continha dados sociodemográficos, informações sobre consumo múltiplo de medicamentos e questões que permitiam investigar, utilizando testes específicos, a presença de alterações cognitivas, depressão, ansiedade e estresse, além do grau de independência funcional.

O exame bucal foi realizado sem nenhum procedimento invasivo, conforme já descrito na literatura [29], utilizando apenas gaze, espátula de madeira e espelho. Para exame do índice CPO-D, foram seguidos os critérios da Organização Mundial da Saúde. Foi realizada uma calibração teórica e prática para o índice CPO-D [30]. Foi calibrado previamente um pesquisador e o resultado do teste Kappa intraexaminador foi 0,95 .

Para o consumo múltiplo de medicamentos foi observada a quantidade diária ingerida por cada participante. A polifarmácia foi definida da seguinte forma: sem polifarmácia (0-2 medicamentos), polifarmácia menor (3-4 medicamentos) e polifarmácia maior ( 5 ou mais medicamentos).

A avaliação da presença de alterações cognitivas foi realizada a partir do teste Mini Exame do Estado Mental (MEEM) adaptado para o Brasil [31]. Essa escala é composta por seis itens com questionamentos simples que permitem avaliar a existência de alteração cognitiva nos indivíduos. Altos escores do MEEM (máximo 30 pontos) indicam melhor capacidade cognitiva, enquanto baixos escores (mínimo 0 pontos) correspondem a um maior grau de comprometimento cognitivo [31]. O idoso que apresentou escore final menor ou igual a 24 foi considerado com comprometimento cognitivo.

A independência funcional foi avaliada a partir da Escala de Katz, que possui seis itens (alimentação, controle de esfíncteres, transferência, higiene pessoal, capacidade para se vestir e tomar banho) com a finalidade de medir o desempenho do indivíduo nas atividades de autocuidado [32].

A depressão foi investigada por meio de dois instrumentos: Inventário de Depressão de Beck (BDI) e Escala de Depressão Geriátrica (EDG). A BDI contém 21 itens: tristeza, pessimismo, sensação de fracasso, insatisfação, sentimento de culpa e de punição, auto depreciação, autoacusações, ideias suicidas, crises de choro, irritabilidade, retração social, indecisão, distorção da imagem corporal, inibição para o trabalho, distúrbio do sono, fadiga, perda de apetite e de peso, preocupação somática e diminuição da libido. Tal instrumento serve para mensurar sintomas e atitudes relacionados à depressão. Os escores indicativos para a depressão são classificados em: normal (0-9), leve (10-15), leve a moderada (16-19), moderada a severa (20-29), severa (30-63) [33].

A EDG é composta por 15 perguntas de fácil entendimento, que avaliam a prevalência de sintomas depressivos na população idosa, podendo ser autoaplicada ou aplicada por um entrevistador treinado. Permite que os entrevistados classifiquem os itens como presentes ou ausentes por meio de respostas dicotômicas sim/não. A amplitude da pontuação é de zero a 15 pontos, sendo classificada como: sem depressão ou depressão mínima (0-5 pontos), depressão leve (6-10 pontos) e depressão grave (11-15 pontos) [34].

A ansiedade foi avaliada pelo instrumento Inventário de Ansiedade de Beck (BAI), que é constituído por 21 itens. O indivíduo deve apontar, em uma escala de quatro pontos, o nível de gravidade do sintoma, sendo que o escore total varia de zero a 63 . O nível de ansiedade é classificado em mínimo (0-7), leve (8-15), moderado (16-25) ou grave (26-63) [35].

O estresse foi avaliado pela escala Inventário de Sintomas de Stress para Adultos de Lipp (ISSL). O ISSL visa a responder três perguntas básicas: se o indivíduo é estressado; em caso positivo, em qual fase (alerta, resistência, quase exaustão ou esgotamento); e se os sintomas físicos ou psicológicos são predominantes. Tal instrumento é composto por três quadros (Q) que se referem às quatro fases do estresse, sendo divididos em sintomas das últimas $24 \mathrm{~h}$ (Q1-fase de alerta), da última semana (Q2-fase de resistência e quase exaustão) e último mês (Q3-fase de exaustão). O diagnóstico positivo é dado a partir da soma dos sintomas de cada quadro do inventário, e ao ultrapassar o número limite em uma fase específica indica-se a ocorrência de estresse e sua fase (Q1>6 sintomas apontados, indica fase de alarme; Q2 $>3$ sintomas, indica fase 
de resistência; Q2 $>9$ sintomas, indica fase de quase exaustão; e Q3>8 sintomas, indica fase de exaustão) [36].

Os dados foram duplamente digitados e validados no programa EpiData 3.1, e a análise estatística utilizo o programa IBM SPSS Statistics (versão 22.0). Foi usado o teste de correlação de Spearman. Para a interpretação e inferência estatística utilizou-se um nível de confiança de $95 \%$ para um erro aleatório máximo até $5 \%(\mathrm{p} \leq 0,05)[27]$.

\section{RESULTADOS}

A amostra foi composta por 37 idosos, sendo 22 mulheres e 15 homens, com idade variando de 63 a

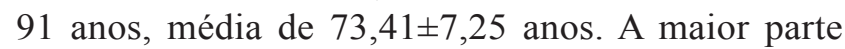
dos participantes possuía residência própria $(94,6 \%)$ e saneamento básico $(94,6 \%)$. O número médio de moradores por residência era de 3,94 (2,23). Instrução, renda e outros dados sociodemográficos estão descritos na Tabela 1.

Na amostra estudada $21,6 \%$ dos idosos apresentaram sintomas indicativos de depressão leve ou moderada de acordo com os resultados da aplicação da BDI. Tais resultados foram confirmados pelos resultados obtidos pela EDG, pela qual foram identificados $18,9 \%$ de idosos com sintomas depressivos (Tabela 1).

Foram detectados pelo BAI 45,9\% dos idosos com ansiedade leve, moderada ou severa, enquanto $54,1 \%$ apresentaram ansiedade ausente ou mínima. Para $81,1 \%$ dos idosos ocorreu a vivência de algum tipo de estresse conforme o ISSL, sendo que para $48,6 \%$ ocorreram manifestações de sintomas na esfera psicossocial. Quanto à avaliação do estado mental pela MEEM, $78,4 \%$ dos idosos apresentavam comprometimento cognitivo. A escala de Katz identificou independência ou ligeira dependência funcional em $81,1 \%$ dos idosos (Tabela 1).

O CPO-D apresentou índice médio de 25,38 55,18. $\mathrm{O}$ consumo médio de medicamentos por idoso foi de $2,92 \pm 2,35$, sendo que a metade $(54,1 \%)$ dos entrevistados apresentou polifarmácia menor, enquanto 18,9\% apresentaram polifarmácia maior (Tabela 1).

Foram encontradas correlações positivas moderadas estatisticamente significantes entre: idade e CPO-D ( $\left.r_{s}=0,351 ; p=0,033\right)$, BDI e BAI $\left(r_{s}=0,590\right.$; $\mathrm{p}=0,001)$, BDI e EDG $\left(\mathrm{r}_{\mathrm{s}}=0,554 ; \mathrm{p}=0,001\right)$, BDI e alarme $\left(r_{s}=0,583 ; p=0,001\right)$, BDI e exaustão $\left(r_{s}=0,567\right.$; $\mathrm{p}=0,001)$, BAI e EDG $\left(\mathrm{r}_{\mathrm{s}}=0,461 ; \mathrm{p}=0,004\right)$, BAI e alarme $\left(r_{s}=0,585 ; p=0,001\right)$, EDG e alarme $\left(r_{s}=0,342\right.$; $\mathrm{p}=0,038)$ e EDG e resistência $\left(\mathrm{r}_{\mathrm{s}}=0,345 ; \mathrm{p}=0,037\right)$ (Tabela 2).
Tabela 1. Características sociodemográficas e fatores psicossociais dos idosos no município de Diamantina, Minas Gerais, Brasil, $2015(n=37)$.

\begin{tabular}{|c|c|c|}
\hline Variável & Média (DP) & n (\%) \\
\hline Idade & $73,41(7,25)$ & \\
\hline $\begin{array}{l}\text { Estrutura familiar } \\
\text { Nuclear } \\
\text { Sem resposta }\end{array}$ & & $\begin{array}{l}13(35,1) \\
24(64,9)\end{array}$ \\
\hline Número de moradores na residência & $3,94(2,23)$ & \\
\hline $\begin{array}{l}\text { Tipo de residência } \\
\text { Própria } \\
\text { Aluguel }\end{array}$ & & $\begin{array}{c}30(81,1) \\
2(5,4)\end{array}$ \\
\hline $\begin{array}{l}\text { Saneamento básico } \\
\text { Sim } \\
\text { Não }\end{array}$ & & $\begin{array}{c}33(89,2) \\
2(5,4)\end{array}$ \\
\hline $\begin{array}{l}\text { Escolaridade } \\
\text { Analfabeto } \\
\text { Ens. Fundamental incompleto } \\
\text { Ens. Fundam. Completo } \\
\text { Ens. Médio incompleto } \\
\text { Ens. Med. Completo } \\
\text { Ensino superior }\end{array}$ & & $\begin{aligned} & (8,1) \\
5 & (13,5) \\
14 & (37,8) \\
& 2(5,4) \\
4 & (10,8) \\
1 & (2,7)\end{aligned}$ \\
\hline $\begin{array}{l}\text { Renda Familiar } \\
<1 \text { salário mínimo } \\
1 \mathrm{a}<2 \text { salários } \\
2 \mathrm{a}<5 \text { salários } \\
5 \mathrm{a}<10 \text { salários }\end{array}$ & & $\begin{aligned} 2 & (5,4) \\
16 & (43,2) \\
18 & (48,6) \\
1 & (2,7)\end{aligned}$ \\
\hline $\begin{array}{l}\text { Escala Katz } \\
\text { Independente } \\
\text { Dependência ligeira } \\
\text { Dependência moderada } \\
\text { Dependência total }\end{array}$ & & $\begin{aligned} 25 & (67.6) \\
5 & (13,5) \\
3 & (8,1) \\
4 & (10,8)\end{aligned}$ \\
\hline $\begin{array}{l}\text { BDI } \\
\text { Sem depressão ou depressão mínima } \\
\text { Depressão leve } \\
\text { Depressão moderada }\end{array}$ & $7,27(5,37)$ & $\begin{aligned} & 29(78,4) \\
& 6(16,2) \\
& 2(5,4)\end{aligned}$ \\
\hline $\begin{array}{l}\text { BAI } \\
\text { Ansiedade mínima } \\
\text { Ansiedade leve } \\
\text { Ansiedade moderada } \\
\text { Ansiedade severa }\end{array}$ & $7,81(7,38)$ & $\begin{aligned} 20 & (54,1) \\
11 & (29,7) \\
5 & (13,5) \\
1 & (2,7)\end{aligned}$ \\
\hline CPO-D & $25,38(5,18)$ & \\
\hline $\begin{array}{l}\text { MEEM } \\
\text { Com comprometimento cognitivo } \\
\text { Sem comprometimento cognitivo }\end{array}$ & $20,39(4,97)$ & $\begin{array}{r}29(78,4) \\
8(21,6)\end{array}$ \\
\hline $\begin{array}{l}\text { EDG } \\
\text { Com depressão } \\
\text { Sem depressão }\end{array}$ & $3,35(2,23)$ & $\begin{array}{r}7(18,9) \\
30(81,1)\end{array}$ \\
\hline $\begin{array}{l}\text { Medicação } \\
\text { Média do número diário de medicamentos } \\
\text { Sem polifarmácia } \\
\text { Polifarmácia menor } \\
\text { Polifarmácia maior }\end{array}$ & $2,92(2,35)$ & $\begin{array}{r}10(27,0) \\
20(54,1) \\
7(18,9)\end{array}$ \\
\hline ISSL alarme & $2,14(2,03)$ & $12(32,4)$ \\
\hline ISSL resistência & $2,05(2,41)$ & $6(16,2)$ \\
\hline ISSL exaustão & $2,46(3,27)$ & $12(32,4)$ \\
\hline Sem estresse & $0,00(0,00)$ & $7(18,9)$ \\
\hline
\end{tabular}

DP, desvio padrão; $\mathrm{BDI}$, inventário de depressão de Beck; BAI, inventário de ansiedade de Beck; CPO-D: índice de dentes cariados, perdidos e obturados; MEEM, mini exame do estado mental; EDG, escala de depressão geriátrica; ISSL, inventário de sintomas de estresse de Lipp. 
Tabela 2. Correlação entre variáveis psicossociais, saúde bucal e idade em 37 idosos de Diamantina, Minas Gerais, Brasil, 2015.

\begin{tabular}{|c|c|c|c|c|c|c|c|c|c|c|c|c|c|c|c|c|}
\hline & \multicolumn{2}{|c|}{ CPOD } & \multicolumn{2}{|c|}{ BDI } & \multicolumn{2}{|c|}{ BAI } & \multicolumn{2}{|c|}{ MEEM } & \multicolumn{2}{|c|}{ Esc. Ger. Depre. } & \multicolumn{2}{|c|}{ ISSL Alarme } & \multicolumn{2}{|c|}{$\begin{array}{c}\text { ISSL } \\
\text { Ressistência }\end{array}$} & \multicolumn{2}{|c|}{ ISSL Exaustão } \\
\hline & $r_{s}$ & $\mathbf{P}$ & $\mathbf{r}_{\mathrm{s}}$ & $\mathbf{P}$ & $r_{s}$ & $\mathbf{P}$ & $r_{s}$ & p & $r_{s}$ & $\mathbf{P}$ & $r_{s}$ & $\mathbf{P}$ & $r_{s}$ & $\mathbf{P}$ & $r_{s}$ & $\mathbf{P}$ \\
\hline Idade & 0,351 & $0,033^{*}$ & 0,233 & 0,164 & $-0,010$ & 0,954 & $-0,463$ & $0,004^{*}$ & 0,071 & 0,677 & 0,027 & 0,876 & 0,030 & 0,859 & 0,070 & 0,682 \\
\hline CPO-D & - & - & 0,173 & 0,306 & 0,210 & 0,213 & $-0,484$ & $0,003^{*}$ & 0,097 & 0,568 & 0,015 & 0,932 & 0,096 & 0,571 & 0,036 & 0,832 \\
\hline BDI & & & - & - & 0,590 & $0,001^{*}$ & $-0,187$ & 0,275 & 0,554 & $0,001^{*}$ & 0,583 & $0,001^{*}$ & 0,690 & $0,001^{*}$ & 0,567 & $0,001^{*}$ \\
\hline BAI & & & & & - & - & $-0,003$ & 0,987 & 0,461 & $0,004^{*}$ & 0,585 & $0,001^{*}$ & 0,710 & $0,001^{*}$ & 0,738 & $0,001^{*}$ \\
\hline MEEM & & & & & & & - & - & 0,203 & 0,236 & 0,001 & 0,996 & $-0,193$ & 0,261 & 0,107 & 0,534 \\
\hline EDG & & & & & & & & & - & - & 0,342 & $0,038^{*}$ & 0,345 & $0,037^{*}$ & 0,379 & 0,021 \\
\hline ISSL alarme & & & & & & & & & & & - & - & 0,615 & $0,001^{*}$ & 0,695 & $0,001^{*}$ \\
\hline ISSL resistência & & & & & & & & & & & & & - & - & 0,802 & $0,001^{*}$ \\
\hline ISSL exaustão & & & & & & & & & & & & & & & - & - \\
\hline
\end{tabular}

Correlação de Spearman. * Correlação estatisticamente significativa.

BDI, Inventário de Depressão de Beck; BAI, Inventário de Ansiedade de Beck; CPO-D, Índice de Dentes Cariados, Perdidos e Obturados; MEEM, Mini Exame do Estado Mental; EDG, Escala de Depressão Geriátrica; ISSL, Inventário de Sintomas de Estresse de Lipp.

Houve correlações negativas moderadas entre idade e MEEM ( $\left.r_{s}=-0,463 ; p=0,004\right)$ e CPO-D e MEEM $\left(r_{s}=-0,484 ; p=0,003\right)$. Houve correlação positiva forte entre BDI e resistência $\left(r_{s}=0,690 ; p=0,001\right)$, BAI e resistência $\left(r_{s}=0,710 ; p=0,001\right)$, BAI e exaustão $\left(r_{s}=0,738 ; p=0,001\right)$, alarme e resistência $\left(r_{s}=0,615\right.$; $\mathrm{p}=0,001)$, alarme e exaustão $\left(\mathrm{r}_{\mathrm{s}}=0,695 ; \mathrm{p}=0,001\right)$ e resistência e exaustão $\left(\mathrm{r}_{\mathrm{s}}=0,802 ; \mathrm{p}=0,001\right)$ (Tabela 2$)$.

\section{DISCUSSÃO}

Vários pesquisadores vêm estudando o perfil cognitivo dos idosos e a relação da presença de queixas simples de memória com o desenvolvimento posterior de demência, o que ainda não está claro. As alterações mais comuns entre os idosos são as que não se caracterizam como demência, só passando a ter significância clínica se acarretarem prejuízo nas atividades diárias do indivíduo [37]. Os idosos são propensos a apresentar desempenho deficitário em testes que avaliam funções executivas e atenção [38]. O presente estudo apontou que apesar da maioria dos idosos terem mostrado declínio de funções cognitivas, uma minoria apresentava dependência funcional moderada ou total. Este resultado sugere que a maioria desses idosos apresentava um declínio funcional da cognição sem evidência de demência.

O comprometimento da funcionalidade do idoso associa-se com restrições na realização de suas atividades de vida diária, sendo esta uma importante adversidade relacionada ao envelhecimento [39]. O presente estudo identificou pela escala Katz que 67,6\% dos idosos eram independentes funcionalmente, não necessitando de ajuda de outra pessoa para a realização de suas atividades da vida diária. De acordo com Cunha et al. [6], os idosos que apresentam algum nível de dependência para realização de atividades diárias tendem a ser mais vulneráveis ao estresse, ansiedade e depressão. Isso porque a diminuição da autonomia do indivíduo idoso pode ser uma experiência de frustração significativa, por representar a perda de uma capacidade individual para realizar e satisfazer as próprias demandas e uma diminuição no controle que o idoso tem sobre si. Esses fatores têm sido mais associados à perda de qualidade de vida na velhice do que os problemas de saúde, que podem ser controlados por medicação [6].

A maior parte dos idosos da amostra estudada fazia uso concomitante de dois ou mais fármacos. Não só as inúmeras patologias e sintomas justificam a polifarmácia; pode ocorrer, inclusive, o uso de fármacos com a mesma indicação terapêutica, pois os idosos tendem a buscar múltiplos atendimentos médicos e, pelo comprometimento de suas habilidades cognitivas, apresentam dificuldade em relatar com exatidão a terapêutica vigente, propiciando que seja prescrito um novo medicamento com efeito semelhante a outro já em uso. Adicionalmente, a automedicação no idoso contribui para a polifarmácia [40-42].

A ansiedade nos idosos está relacionada às limitações vivenciadas na velhice, na maioria das vezes interpretadas como ameaçadoras. Pessoas ansiosas tendem a antecipar uma futura inabilidade e questionar sua atual capacidade intelectual, antes mesmo de qualquer comprometimento, interferindo na atenção seletiva e na codificação de informações da memória e prejudicando a compreensão e o raciocínio [43]. $\mathrm{O}$ presente estudo mostrou que todos os idosos 
apresentavam algum grau de ansiedade, sendo que mais da metade dessa amostra apresentava ansiedade mínima, considerada natural e adaptativa. Entretanto, destaca-se o índice de $45,9 \%$ de idosos com ansiedade (aferida pelo BAI) de leve a severa, tendo apresentado correlação com estresse do tipo alerta, resistência e exaustão.

Além disso, as correlações entre depressão (utilizando BDI e EDG) e ansiedade (utilizando BAI) encontradas nesse estudo também corroboram com achados anteriores sobre a associação entre ansiedade e depressão [43]. Alguns fatores de risco que produzem estresse ao indivíduo idoso e estão relacionados à ocorrência tanto de ansiedade quanto de depressão são a diminuição da capacidade funcional, a falta de suporte por parte da família, menor satisfação com as relações pessoais, sofrimento físico e psíquico devido ao agravamento de doenças e incapacidades, morte de familiares e amigos, acesso restrito a ambientes e atividades anteriormente satisfatórios, dentre outros fatores [11-13]. Vale ressaltar a importância do diagnóstico em saúde mental e acompanhamento geriátrico com equipe multidisciplinar para que seja garantido o cuidado global da saúde dessa população.

A depressão é o problema de saúde mental mais comum na terceira idade, tendo impacto negativo em todos os aspectos da vida, sendo assim de grande relevância na saúde pública [44]. Entretanto, ainda é comum a atribuição errônea dos sintomas depressivos ao processo de envelhecimento normal, por parte do próprio idoso, de seus familiares e de alguns profissionais de saúde [3]. Neste estudo, foi encontrada uma prevalência expressiva de indicadores para depressão nos idosos estudados, em comparação à prevalência estimada em outros estudos: $21,6 \%$ de idosos com depressão leve ou moderada pelo BDI e $18,9 \%$ com depressão pela EDG, em comparação a $15 \%$ no estudo de Cunha et al. [9]. Em alguns estudos verificou-se que as altas prevalências de depressão podem ser decorrentes do fato da amostra ser composta por idosos hospitalizados, o que não foi a situação da presente amostra [39,45-47].

É evidente a correlação entre escore de MEEM, baixa escolaridade e presença de sintomas depressivos. Como neste estudo foi expressiva a presença de indicadores de depressão justifica-se o resultado abaixo do ponto de corte no MEEM. A depressão prejudica algumas funções cognitivas, de forma que quando o idoso apresenta sintomas depressivos, ele muito provavelmente terá uma baixa pontuação pelo MEEM, não significando necessariamente que apresente demência.

A grande maioria dos entrevistados foi classificada como estressada (resistência, quase exaustão ou exaustão). Provavelmente, os idosos podem estar submetidos a fatores como burocracia, dificuldades financeiras, problemas com o atendimento de saúde, morte de amigos e familiares e discriminação social, que os tornam cada vez mais suscetíveis ao estresse [11-13].

Assim como em outros estudos realizados no Brasil $[48,49]$, a alta taxa de edentulismo encontrada neste estudo contribuiu para um alto índice CPO-D. Isto mostra que o modelo de saúde bucal do nosso país ainda é baseado em ações terapêuticas com menor custo, o que leva os profissionais a recorrerem frequentemente às extrações dentárias [50]. Dessa forma, faz-se necessária a mudança da política de saúde pública atual para que sejam priorizadas a prevenção e a promoção de saúde bucal [27].

O presente estudo piloto, apesar de ter utilizado inquérito domiciliar com amostra probabilística e bem conduzido, apresenta algumas limitações. Por exemplo, a impossibilidade da identificação temporal dos fatores estudados e a ausência de análises estatísticas mais robustas. É importante salientar que a idade da população em estudo pode ser considerada como um fator de confundimento entre as correlações aqui estabelecidas. Por exemplo, a correlação entre saúde bucal e saúde mental pode ser devida ao fato de que as duas variáveis se relacionam com idade. Este estudo se beneficiaria de uma análise multivariada, tendo em vista as muitas variáveis de interesse. Entretanto, análises de regressão para determinar quais variáveis poderiam melhor explicar os desfechos dos questionários utilizados não puderam ser concluídas devido ao pequeno número amostral deste estudo piloto.

Pelo presente estudo conclui-se que a saúde bucal apresentou alto índice CPO-D em idosos cadastrados nas Estratégias Saúde da Família de Diamantina, MG. Saúde bucal, ansiedade, depressão, estresse e medicação foram fatores diretamente correlacionados nos idosos estudados. Os profissionais de saúde devem estar atentos não apenas aos eventos clínicos do envelhecimento, mas também à presença de fatores psicossociais como os detectados neste estudo. 
NOTA

Declaração de conflitos de interesse

Os autores dclaram não haver conflitos de iteresse relevantes ao conteúdo deste estudo.

\section{REFERÊNCIAS}

1. Lima-Costa MF, Veras R. Saúde pública e envelhecimento. Cad Saude Publica. 2003 May-June;19(3):701, 700.

2. United Nations. World population prospects: the 2002 revision [Internet]. New York: United Nations, 2003 [cited 2015 Aug 22]. Available from: http://esa.un.org/unpd/wpp/

3. Frank MH, Rodrigues NL. Depressão, Ansiedade, Outros Transtornos Afetivos e Suicídio. In: Freitas EV, Py L, Neri AL, Cançado FLX, Gorzoni ML, Rocha SM, organizadores. Tratado de Geriatria e Gerontologia. Rio de Janeiro: Guanabara Koogan; 2002. p. $376-87$.

4. Fagundes SD, Silva MT, Thees MF, Pereira MG. Prevalence of dementia among elderly Brazilians: a systematic review. Sao Paulo Med J. 2011 Jan 6;129(1):46-50. http://dx.doi.org/10.1590/S1516-31802011000100009

5. Eulálio MC, Andrade TF, Melo RLP, Neri AL. A estrutura latente da depressão em idosos: uma análise taxométrica. Cad Saude Publica. 2015 Mar;31(3):555-64. http://dx.doi.org/10.1590/0102-311X00028914

6. Cunha R V, Bastos GAN, Duca GFD. Prevalência de depressão e fatores associados em comunidade de baixa renda de Porto Alegre, Rio Grande do Sul. Rev Bras Epidemiol. 2012;15:346-54. http://dx.doi.org/10.1590/S1415-790X2012000200012

7. Silva ER, Sousa ARP, Ferreira LBF, Peixoto HM. Prevalência e fatores associados à depressão entre idosos institucionalizados: subsídio ao cuidado de enfermagem. Rev Esc Enferm USP. 2012;46:1387-93. http://dx.doi.org/10.1590/S0080-62342012000600015

8. Carreira L, Botelho MR, Matos PCB, Torres MM, Salci MA. Prevalência de depressão em idosos institucionalizados. Rev Enferm UERJ. 2011;19:268-73.

9. Blazer D. Depressão em idosos. 3ำ ed. São Paulo: Andrei; 2003.

10. Stella F. Ansiedade no idoso. In: Forlenza OV, organizador. Psiquiatria geriátrica: do diagnóstico precoce à reabilitação. São Paulo: Editora Atheneu; 2007. p. 97-106.

11. Carl JR, Soskin DP, Kerns C, Barlow DH. Positive emotion regulation in emotional disorders: a theoretical review. Clin Psychol Rev. 2013 Apr;33(3):343-60. http://dx.doi.org/10.1016/j.cpr.2013.01.003

12. Wang JJ, Snyder M, Kaas M. Stress, loneliness, and depression in Taiwanese rural community-dwelling elders. Int J Nurs Stud. 2001 June;38(3):339-47. http://dx.doi.org/10.1016/S0020-7489(00)00072-9

13. Xavier FM, Ferraz MP, Trenti CM, Argimon I, Bertolucci PH, Poyares D, Moriguchi EH. Transtorno de ansiedade generalizada em idosos com oitenta anos ou mais. Rev Saude Publica. 2001 Jun;35(3):294-302. http://dx.doi.org/10.1590/S0034-89102001000300013

14. Rait G, Fletcher A, Smeeth L, Brayne C, Stirling S, Nunes M, Breeze E, Ng ES, Bulpitt CJ, Jones D, Tulloch AJ. Prevalence of cognitive impairment: results from the MRC trial of assessment and management of older people in the community. Age Ageing. 2005 May;34(3): 242-8. http://dx.doi.org/10.1093/ageing/afi039

15. Verbrugge LM1, Jette AM. The disablement process. Soc Sci Med. 1994 Jan;38(1):1-14. http://dx.doi.org/10.1016/0277-9536(94)90294-1

16. Linjakumpu T1, Hartikainen S, Klaukka T, Veijola J, Kivelä SL, Isoaho R. Use of medications and polypharmacy are increasing among the elderly. J Clin Epidemiol. 2002 Aug;55(8):809-17. http://dx.doi.org/10.1016/S0895-4356(02)00411-0

17. Gagliardi AMZ, Almada Filho CM. Infecções no idoso frágil. In: Prado FC, Ramos J, Valle JR, editores. Atualização terapêutica. São Paulo: Artes Médicas; 2003. p. 577-80.

18. Lebrão ML, Duarte YAO, Santos JLF, Laurenti R. Evolução nas condições de vida e saúde da população idosa do Município de São Paulo. Search Results. São Paulo Perspec. 2008;22(2):30-45.

19. Gorzini ML, Passareli MCG. Farmacologia e terapêutica na velhice. In: Freitas EV, Py L, editores. Tratado de Geriatria e Gerontologia. $2^{a}$ ed. Rio de Janeiro: Guanabara Koogan; 2006. p.942-8.

20. Rozenfeld S. Prevalência, fatores associados e mau uso de medicamentos entre idosos: uma revisão. Cad Saude Publica. 2003 MayJune;19(3):717-24. http://dx.doi.org/10.1590/S0102-311X2003000300004

21. Iliffe S, Haines A, Gallivan S, Booroff A, Goldenberg E, Morgan P.Assessment of elderly people in general practice. 2. Functional abilities and medical problems. Br J Gen Pract. 1991 Jan;41(342):13-5.

22. Veehof LJ, Stewart R, Haaijer-Ruskamp FM, Meyboom-de Jong B. Chronic polypharmacy in one-third of the elderly in family practice. Ned Tijdschr Geneeskd. 1999 Jan 9;143(2):93-7.

23. Veehof L, Stewart R, Haaijer-Ruskamp F, Jong BM. The development of polypharmacy. A longitudinal study. Fam Pract. 2000 June;17(3): 261-7. http://dx.doi.org/10.1093/fampra/17.3.261

24. Hanlon JT, Schmader KE, Ruby CM, Weinberger M. Suboptimal prescribing in older inpatients and outpatients. J Am Geriatr Soc. 2001 Feb;49(2):200-9. http://dx.doi.org/10.1046/j.1532-5415.2001.49042.x

25. Jörgensen TM, Isacson DG, Thorslund M. Presciption drug use among ambulatory elderly in a Swedish municipality. Ann Pharmacother. 1993 Sept;27(9):1120-5.

26. Linjakumpu T, Hartikainen S, Isoaho R, Kivelä S-L. Polypharmacy and the use of psychotropics and analgesic drugs among the communitydwelling elderly. Gerontologia. 2001;15:117-24.

27. Menezes-Silva R, de Oliveira DWD, Sá-Pinto AC, Biscaro PCB, Orti NP, Ramos-Jorge ML. Inquérito epidemiológico em saúde bucal e fatores psicossociais em idosos - um estudo piloto. Sci Med. 2015;25(2):ID20918. http://dx.doi.org/10.15448/1980-6108.2015.2.20918 
28. Hertzog MA. Considerations in Determining Sample Size for Pilot Studies. Res Res Nurs Health. 2008 Apr;31(2):180-91. http://dx.doi. org/10.1002/nur.20247

29. Cypriano S, Pecharki GD, de Sousa Mda L, Wada RS. A saúde bucal de escolares residentes em locais com ou sem fluoretação nas águas de abastecimento público na região de Sorocaba, São Paulo, Brasil. Cad Saude Publica. 2003 July-Aug;19(4):1063-71. http://dx.doi. org/10.1590/S0102-311X2003000400028

30. Organização Mundial de Saúde. Levantamentos básicos em saúde bucal. 4ํㅡㄹ. São Paulo: Santos; 1999.

31. Folstein MF, Folstein SE, McHugh PR. "Mini-Mental State": a practical method for grading the cognitive state of patients for the clinician. J Psychiatr Res. 1975 Nov;12(3):189-98. http://dx.doi.org/10.1016/0022-3956(75)90026-6

32. Evans BC, Crogan NL. Building a scientific base for nutrition care of Hispanic nursing home residents. Geriatr Nurs. 2006 SeptOct;27(5):273-9. http://dx.doi.org/10.1016/j.gerinurse.2006.08.006

33. Gorenstein C, Andrade L. Validation on of a portuguese version of the beck depression inventory and the state-trait anxiety inventory in Brazilian Subjects. Braz J Med Biol Res. 1996 Apr;29(4):453-7.

34. Alvarenga MRM, Oliveira MAC, Faccenda O. Sintomas depressivos em idosos: análise dos itens da Escala de Depressão Geriátrica. Acta Paul Enferm. 2012;25(4):497-503. http://dx.doi.org/10.1590/S0103-21002012000400003

35. Cunha JA. Manual da versão em português das escalas Beck. São Paulo: Casa do Psicólogo; 2001.

36. Lipp MEN. Manual do inventário de sintomas de estresse para adultos de LIPP. 3ํㅡ ed. São Paulo: Casa do Psicólogo; 2005.

37. Canineu PR, Samara AB, Stella F. Transtorno cognitivo leve. In: Freitas EV, Py L, Cançado FAX, Doll J, Gorzoni ML. Tratado de geriatria e gerontologia. 2a ed. Rio de Janeiro: Guanabara-Koogan; 2006. p. 253-9

38. Swainson R, Hodges JR, Galton CJ, Semple J, Michael A, Dunn BD, Iddon JL, Robbins TW, Sahakian BJ. Early detection and differential diagnosis of Alzheimer's disease and depression with neuropsychological tasks. Dement Geriatr Cogn Disord. 2001 July-Aug;12(4):265-80. http://dx.doi.org/10.1159/000051269

39. Carvalho Filho ET, Papaléo Netto M. Geriatria: fundamentos, clínica e terapêutica. $2^{\text {a }}$ ed. São Paulo: Atheneu; 2006.

40. Roesler e Silva E, Sousa AR, Ferreira LB, Peixoto HM. Prevalência e fatores associados à depressão entre idosos institucionalizados: subsídio ao cuidado de enfermagem. Rev Esc Enferm USP. 2012 Dec;46(6):1387-93. http://dx.doi.org/10.1590/S0080-62342012000600015

41. Teixeira, JJV, Lefèvre F, Castro, LLC, Spinola AWP. Levantamento bibliográfio sobre o cumprimento da prescrição medicamentosa por idosos no Brasil, segundo resumos de congressos. Arq Geriatr Gerontol. 2000;4:63-7.

42. Ramos Cede-o AM, Milián Vázquez PM, Fonseca León JL, Quirós Enríquez M. Determinación de polifarmacoterapiaen pacientes geriátricos de um consultoriodel médico de lafamilia em Cienfuegos. Rev Cubana Farm 2000;34(3):170-4.

43. Oliveira KL, dos Santos AAA, Cruvinel M, Néri AL. Relação entre ansiedade, depressão e desesperança entre grupos de idosos. Psicol Estud. 2006;11:351-9. http://dx.doi.org/10.1590/S1413-73722006000200014

44. Canadian Coalition for Seniors' Mental Health. The assessment and treatment of depression. Toronto: CCSMH; 2006.

45. Siqueira GR, Vasconcelos DT, Duarte GC, Arruda IC, Costa JAS, Cardoso RO. Análise da sintomatologia depressiva nos moradores do Abrigo Cristo Redentor através da aplicação da Escala de Depressão Geriátrica (EDG). Ciênc Saúde Colet. 2009;14:253-9. http://dx.doi org/10.1590/S1413-81232009000100031

46. McDougall FA, Matthews FE, Kvaal K, Dewey ME, Brayne C. Prevalence and symptomatology of depression in older people living in institutions in England and Wales. Age Ageing. 2007 Sept;36(5):562-8. http://dx.doi.org/10.1093/ageing/afm111

47. Porcu M, Scantamburlo VM, Albrecht NR, Silva SP, Vallim FL, Araújo CR, Deltreggia C, Faiola RV. Estudo comparativo sobre a prevalência de sintomas depressivos em idosos hospitalizados, institucionalizados e residentes na comunidade. Acta Sci Health Sci. 2002; 24:713-7.

48. Moreira RS, Nico LS, Tomita NE, Ruiz T. Oral health of Brazilian elderly: a systematic review of epidemiologic status and dental care access. Cad Saude Publica. 2005 Nov-Dec;21(6):1665-75. http://dx.doi.org/10.1590/S0102-311X2005000600013

49. Hugo FN, Hilgert JB, de Sousa Mda L, da Silva DD, Pucca GA Jr. Correlates of partial tooth lossandedentulism in the Brazilian elderly. Community Dent Oral Epidemiol. 2007 June;35(3):224-32. http://dx.doi.org/10.1111/j.0301-5661.2007.00346.x

50. Ulinski KG, do Nascimento MA, Lima AM, Benetti AR, Poli-Frederico RC, Fernandes KB, Fracasso ML, Maciel SM. Factors related to oral health-related quality of life of independent brazilian elderly. Int J Dent. 2013;2013:705047. http://dx.doi.org/10.1155/2013/705047 C 